\title{
Comparison of effectiveness of ormeloxifene with norethisterone in perimenopausal DUB treatment
}

\author{
Surabhi HD ${ }^{1}$, Chandra Mouli $\mathrm{A}^{2 *}$ \\ ${ }^{\mathbf{1}}$ Assistant Professor, ${ }^{\mathbf{2}}$ Associate Professor, ${ }^{\mathbf{1 - 2}}$ Department of Obstetrics and Gynaecology, ${ }^{\mathbf{1}, \mathbf{2}}$ East Point College of Medical Sciences and \\ Research Centre, Bangalore, India \\ *Corresponding Author: Chandra Mouli A \\ Email: mouliaruna2@gmail.com
}

Received: $20^{\text {th }}$ December, 2018

Accepted: $22^{\text {nd }}$ April, 2019

\begin{abstract}
Introduction: Dysfunctional Uterine Bleeding, is Abnormal uterine bleeding in the absence of organic, systemic, iatrogenic cause. ${ }^{1}$ Norethisterone acetate is commoly used progesterone derivative for management. ${ }^{2}$ Ormeloxifene, a selective estrogen receptor modulator is an another alternate treatment for DUB. ${ }^{3}$ Study was conducted at MVJ Medical College Bangalore.

Objective: to compare treatment efficacy and usefulnes of Ormeloxifene and oral progesterone in DUB treatment.

Materials and Methods: Total of 60 perimenopausal women with DUB were enrolled in our prospective study. They were randomly assigned to group A and group B.

Group A: 30 women with DUB were treated with $60 \mathrm{mg}$ Ormeloxifene two times a week for 3 months, 60mg weekly once for next 3 months.

Group B: Norethisterone $5 \mathrm{mg}$ two times a day for 21 days for 6 cycles.

Results: In our study even though Norethistrone group showed significant reduction in blood loss and also increase in haemoglobin level, these results were significantly less when compared with Ormeloxifene group.

In addition Ormeloxifene group showed significant reduction in endometrial thickness, which was not significantly reduced in Norethisterone group. As a result of significant reduction of endometrial thickness among the women treated with Ormeloxifene.

Conclusion: In the study Ormeloxifene was found to be effective than Norethisterone in reducing blood loss, improving Haemoglobin level and reducing endometrial thickness for treating DUB patients. Also compliance of the patients was good because drug was well tolerated, convenient dosage schedule for treatment of DUB.
\end{abstract}

Keywords: Ormeloxifene, Norethisterone, Haemoglobin, Endometrial thickness, PBAC.

\section{Introduction}

Disorders of menstruation account for the most common reason for a gynaecological consultation among women of reproductive age group DUB is one such condition most commonly affecting women from extremes of reproductive age group. ${ }^{1}$ Dysfunction uterine bleeding is abnormal uterine bleeding during menstrual cycle in the absence of organic pelvic pathology. ${ }^{2}$

Blood loss more than $80 \mathrm{ml}$ during the menstrual cycle affects upto $30 \%$ of reproductive aged women and accounts for $70 \%$ of all gynaecologic visits by perimenopausal women. ${ }^{4}$ Dysfunction of HPO axis leads to anovulatory DUB in this age group with major implications on quality of life and health care costs. ${ }^{3}$ Though hysterectomy is a suitable treatment modality, long term complication like premature ovarian insufficiency, urinary dysfunction has raised concerns. ${ }^{4}$ Hence a majority of women are looking forward to an effective conservative medical therapy.

Various pharmacological agents used in treatment of DUB are combined oral, progestogens, danazol, Gn RH agonists, PGsynthetase inhibitors, antifibrinolytics and ethamsylate. 5

A reliable drug should meet requirements such as drug should be effective, easy to take, minimal cost and side effects and have longest safety margin. ${ }^{5}$ Norethisterone is a progestogen widely used for treating DUB. Since it is a hormonal preparation, side effects such as stroke, heart disease, breast cancer, dementia, fluid retention, break through bleeding, spotting has been reported.

As the name suggests SERM is a class of drug which has estrogenic action in some parts of the body and anti estrogenic in some parts. ${ }^{6}$ It has anti estrogenic effect on uterine and breast tissue and estrogenic effect on vagina, CVS and CNS. One of the drug that belongs to this group is Ormeloxifene. It is also popularly known as centchroman, which is a non-steroidal, non- hormonal oral contraceptive taken once in a week when used as contraceptive. ${ }^{7}$ In the treatment of DUB, the standard dose is $60 \mathrm{mg}$ orally two times a week for a period of 3 months followed by once a week in next 3 months. ${ }^{8}$

Since its usage from early 1990's in India, as a contraceptive medication, it has also been useful in treatment of DUB with a convenience in dosing pattern improving patient compliance. ${ }^{9}$

Nausea, headache, weight gain, delayed period are few adverse effect noted on taking the drug. Since it has less uterine side effects, prevents bone loss, no risk of breast cancer, has positive effect on lipids and CVS and maintains cognitive function of brain, it is the ideal drug to be used during perimenopausal age group. ${ }^{9}$

\section{Materials and Methods}

The study population comprised of 60 perimenopausal women with complaints of excessive bleeding during cycles 
diagnosed with DUB attending OPD of OBG dept of MVJ $\mathrm{MC}$ and RH.

They were randomly allocated to 2 groups of 30 subjects each

Group 1: Treated with $60 \mathrm{mg}$ Ormeloxifene, two times a week for 3 months, $60 \mathrm{mg}$ of Ormeloxifene weekly once for next 3 months.

Group 2: Norethisterone $5 \mathrm{mg}$ BD for 21 days for 6 cycles. Primary outcome was measured by reduction in blood loss, by using PBAC score, rise in $\mathrm{Hb}$ and decreased in ET.

\section{Inclusion Criteria}

Perimenopausal women between 40 to 50yrs of age with complaints of heavy menstrual blood loss.

\section{Exclusion Criteria}

Haemodynamically unstable patients, organic pelvic pathology.

\section{Methodology}

Women with heavy menstrual bleeding were subjected to detailed history taking and physical examination, clinical examination includes per speculum, per vaginal exam to exclude organic pelvic pathology. Investigation like CBC, Thyroid profile, coagulation profile, pap smear, pelvic USG for ET and to rule out pelvic pathology were done. After taking informed consent 30 women treated with $60 \mathrm{mg}$ Ormeloxifene and 30 women treated with $5 \mathrm{mg}$ Norethisterone.

Menstrual blood loss was measured by pictorial blood loss chart, endometrial thickness measured using ultrasound, haemoglobin measure by laborartory method.

In order to standardise the PBAC, all subjects candidate for the study were instructed to use same brand of sanitary napkin. PBAC score was calculated by taking in to account number of pads used, number and size of clots passed, soakage of pads. ${ }^{10}$

Table 1: Pictorial blood loss assessment chart (PBAC)

\begin{tabular}{|l|l|c|}
\hline & & Score \\
\hline \multirow{3}{*}{ PADS } & lightly soiled & 1 \\
\cline { 2 - 3 } & moderately soiled & 5 \\
\cline { 2 - 3 } & saturated & 20 \\
\hline \multirow{2}{*}{ CLOTS } & small (smaller than a rupee coin) & 1 \\
\cline { 2 - 3 } & large (larger than a rupee coin) & 5 \\
\hline
\end{tabular}

Each patient in the study was followed up every month during the treatment period. PBAC score was calculated in every cycle. Side effect of the drug was also reviewed. Measures of primary outcome were noted by decreased blood loss during menstrual cycles. This was reflected by reduced PBAC score, increase in $\mathrm{Hb} \%$, reduction in endometrial thickness.

\section{Results}

Women were divided into two groups with similarity related to duration of symptoms, parity, age and socioeconomic status. Women in both groups were compared with pretreatment mean of haemoglobin level, endometrial thickness and PBAC (Table 2).

Table 2: Parameters before the Start of Therapy

\begin{tabular}{|c|c|c|}
\hline Parameters & Group 1 & Group 2 \\
\hline PBAC Score (mean) & 294.33 & 288.56 \\
\hline Hb gm\% (mean) & 9.02 & 9.12 \\
\hline Mean Endometrial Thickness (mm) & 7.4 & 6.9 \\
\hline
\end{tabular}

Group A: Reported with pretreatment mean $\mathrm{Hb}$ of $9.02 \mathrm{~g} \%$ with $7.4 \mathrm{~mm}$ of mean endometrial thickness and mean PBAC of 294.33score.

Group B: Reported with mean $\mathrm{Hb} 9.12 \mathrm{~g} \%$ with $6.9 \mathrm{~mm}$ of mean endometrial thickness and mean PBAC of 288.56 score.

At the end of study period the mean PBAC was 64.11 in group $\mathrm{A}$, which accounts for about $78.21 \%$ reduction of blood loss during menstruation as compared with mean PBAC of 103.55 in group B which accounts for $60.64 \%$ reduction in blood loss during menstrual cycle (Table 3 ). There was statistically significant reduction in PBAC in both groups with $\mathrm{p}$ value less than 0.001. Group A showed much more significant reduction in PBAC as compared with group B.

Table 3: PBAC chart:

\begin{tabular}{|c|c|c|c|c|}
\hline & Pre Treatment PBAC & Posttreatment PBAC & Mean difference & P \\
\hline Ormeloxifene & 294.33 & 64.11 & 230.22 & $<0.001$ \\
\hline Norethisterone & 288.56 & 103.55 & 185.01 & $<0.001$ \\
\hline
\end{tabular}

The group A women reported of mean increase of $2.49 \mathrm{~g} \%$ of haemoglobin when group B women reported mean increase of $1.31 \mathrm{~g} \%$ of haemoglobin. The haemoglobin reported in group A at the end of treatment was $11.51 \mathrm{~g} \%$ as compared with group B women who reported $10.43 \mathrm{~g} \%$ (Table 4). Haemoglobin levels reported statistically increase in both groups with $\mathrm{p}$ value less than 0.001. Group A reported more rise in haemoglobin when compared with group B. 
Table 4: Haemoglobin chart

\begin{tabular}{|c|c|c|c|c|c|}
\hline & Pre-treatment Hb\% & Post-treatment Hb\% & Mean difference & $\mathbf{t}$ & $\mathbf{P}$ \\
\hline Ormeloxifene & 9.02 & 11.51 & 2.49 & 11.75 & $<0.001$ \\
\hline Norethisterone & 9.12 & 10.43 & 1.31 & 4.157 & $<0.001$ \\
\hline
\end{tabular}

Mean reduction of $2.3 \mathrm{~mm}$ of endometrial thickness was documented in group A women when compared with group B women who documented with mean reduction of $1.2 \mathrm{~mm}$ of endometrial thickness (Table -5 ). The reduction of endometrial thickness with $\mathrm{p}$ value less then 0.001 was statistically significant in group A. Group B had p value of 0.023 which was not statistically significant.

Table 5: Reduction in Endometrial Thickness (ET-mm)

\begin{tabular}{|c|c|c|c|c|c|}
\hline & Pretreatment ET & Post treatment ET & Mean difference & $\mathbf{t}$ & $\mathbf{p}$ \\
\hline Ormeloxifene & 7.4 & 5.1 & 2.3 & 4.7 & $<0.001$ \\
\hline Norethisterone & 6.9 & 5.7 & 1.2 & 1.7 & 0.109 \\
\hline
\end{tabular}

\section{Discussion}

DUB is the commonest condition for which women have to attend the gynaecological OPD. Since it is one of the conditions which affect the quality of life, there is urgent need for treating women with DUB.

Various modes of management has been practiced like.

1. Medical management with NSAIDs, progesterones, antifibrinolytics, contraceptive pills.

2. Levonorgestrel releasing intra uterine device

3. Surgery

Endometrial ablation, hysterectomy

The efficacy and safety of Ormeloxifene was compared with Norethisteone in the present study which was done prospectively. Sixty perimenopausal women with DUB were enrolled in our study. They were randomly grouped in two groups.

Group A: women were treated with $60 \mathrm{mg}$ with Ormeloxifene two times a week for three months, followed by weekly once for next three months.

Group B: Norethisterone 5mg twice daily for 21 days for 6 cycles.

The treatment efficacy of both the drugs was compared using following parameters.

1. Pretreatment blood loss during menstrual cycle assessed by PBAC score compared with post treatment blood loss.

2. Pretreatment haemoglobin level compared with post treatment haemoglobin level.

3. Pretreatment endometrial thickess compared with post treatment endometrial thickness.

Our study documented reduction of blood loss in group A (Ormeloxifene) about $78.21 \%$. Blood loss score of 294.11 pretreatment was reduced to 64.11 score. Group B (Norethisterone) reported about $60.64 \%$ reduction of blood loss from 288.56 pretreatment score to 113.55 .

Study conducted by Jacob KJ reported reduction of blood loss by $74.71 \%$ in Ormeloxifene group as compared with Norethisterone group sowed $55.87 \%$ reduction in blood loss. ${ }^{11}$

Study conducted by Jimitkumar JC reported reduction of blood loss by $56.8 \%$ in Ormeloxifene group as compared with OCP group showed 38\% reduction in blood loss score. $^{12}$

Study conducted by Shazia AK reported reduction of blood loss by $83.22 \%$ with Ormeloxifene treatment. ${ }^{13}$

In our study rise of haemoglobin by $2.49 \mathrm{~g} \%$ in group A (Ormeloxifene) as compared to group B (Norethisterone) documented $1.31 \mathrm{~g} \%$ improvement in haemoglobin level.

Study conducted by Jacob KJ reported rise of haemoglobin by $1.39 \mathrm{~g} \%$ with Ormeloxifene as compared with $0.41 \mathrm{~g} \%$ rise of haemoglobin with Norethisterone group. ${ }^{11}$

Study conducted by Jimitkumar JC reported improvement of haemoglobin by $3.1 \mathrm{~g} \%$ with Ormeloxifene as compared with $2.3 \mathrm{~g} \%$ improvement of haemoglobin with OCP group. ${ }^{12}$

Study conducted by Shazia AK reported improvement of haemoglobin by $1.3 \mathrm{~g} \%$ with Ormeloxifene treatment. ${ }^{13}$

Our study documented reduction of endometrial thickness by $2.3 \mathrm{~mm}$ (mean) in group A (Ormeloxifene) and group B (Norethisterone) reported $1.2 \mathrm{~mm}$ (mean) reduction.

Study conducted by Jacob KJ reported Ormeloxifene treatment reduced endometrial thickness by $2.47 \mathrm{~mm}$ and Norethsterone by $0.8 \mathrm{~mm}$ reduction in endometrial thickness. ${ }^{11}$

Study conducted by Shazia AK reported reduction in endometrial thickness by $2.6 \mathrm{~mm}$ with Ormeloxifene treatment ${ }^{13}$.

In our study even though Norethistrone group showed significant reduction in blood loss and also increase in haemoglobin level, these results were significantly less when compared with Ormeloxifene group.

In addition Ormeloxifene group reported significant reduction in endometrial thickness, which was not significantly reduced in Norethisterone group.

In our study Ormeloxifene found more effective in treatment of DUB than Norethisterone with good patient compliance, well tolerated drug and convenient dosage schedule. Hence our study concludes Ormeloxifene as better choice over Norethisterone in treatment of DUB. 


\section{Conclusion}

As the study documents significant decrease in PBAC and increase in haemoglobin levels with both the drugs, Ormeloxifene has better results. Ormeloxifene in addition had significant reduction of endometrial thickness. Even though the treatment with Norethisterone had good results, our study concludes that Ormeloxifene as more effective drug for treatment of DUB. Patient compliance was good as the dose schedule for treatment was convenient and the drug was well tolerated.

\section{Conflict of Interest: None.}

\section{References}

1. National Institute for Health and Clinical Excellence. Heavy Menstrual Bleeding. 2007. Available from URL: http://www.nice.org.uk/cg004.

2. Agarwal.N, S. Singh. The efficiency and safety of ormeloxifene in dysfunctional uterine bleeding. IOSR J Pharm Biol Sci 2013;5(5):18-21.

3. Hallberg L, Hodgahl AM, Nilsson L, Rybo G. Menstrual blood loss - A population study Variation at different ages and attempts to define normality. Acta Obstet Gynecol Scand 1966;45:320-51

4. Frager IS, Jansen RPS, Lobo RA, Whitehead MI. Estrogens and Progestogens in clinical practice. In: Dysfunctional uterine bleeding. Churchill Living Stone Publication 1998:419-37.

5. Pitkin J. Dysfunctional uterine bleeding. $B M J$ 2007;334(7603):1110-1.
6. Studd J. Progress in obstetrics and gynecology. In: Current minimal access techniques for dysfunctional uterine bleeding. Churchill Living Stone, 2003;15:259-72.

7. National Health Committee. Guidelines for the management of heavy menstrual bleeding. 1998 May, updated in 2001.

8. Lethaby A, Irvine GA, Cameron IT. Cyclical progestogens for heavy menstrual bleeding [Online]. Available from: Cochrane Database of systematic reviews; 2008.

9. Osborne CK, Zhao H, Fuqua SA. Selective estrogen receptor modulators: structure, function, and clinical use. J Clin Oncol 2000;18:3172-86.

10. Higham JM, O'Brien PMS, Shaw RW. Assessment of menstrual blood loss using a pictorial chart. Br J Obstet Gynaecol 1990;97:734-9.

11. Jacob KJ, Mini, Deepak AV. A Comparative study on the effectiveness of ormeloxifene versus norethisterone in the management of perimenopausal dysfunctional uterine bleeding. IAIM 2015;2(7):87-92.

12. Chhatrala JJ, Chawada R, Saini HB. Comparative study between Ormeloxifene and oral contraceptive pills in the treatment of dysfunctional uterine bleeding. IJRCOG 2015;4(2):366-9.

13. Shazia AK, Manzoor M, Abdullah A, Banoo A. Efficacy and safety of Ormeloxifene in the treatment of dysfunction uterine bleeding. IOSR-JDMS 2014;13(6);39-42.

How to cite this article: Surabhi HD, Chandra MA, Comparison of effectiveness of ormeloxifene with norethisterone in perimenopausal dub treatment. Indian $J$ Obstet Gynecol Res 2019;6(2):137-140. 